Research Paper

\title{
Epstein-Barr virus miR-BART1-3p suppresses apoptosis and promotes migration of gastric carcinoma cells by targeting DAB2
}

\author{
Kyoungmi Min, Jun Yeob Kim, Suk Kyeong Lee ${ }^{凶}$ \\ Department of Medical Life Sciences, Department of Biomedicine \& Health Sciences, College of Medicine, The Catholic University of Korea, Seoul, Republic of \\ Korea \\ $\square$ Corresponding author: Suk Kyeong Lee, Mailing address: Department of Medical Life Sciences, Department of Biomedicine \& Health Sciences, College of \\ Medicine, The Catholic University of Korea, 222 Banpo-daero, Seocho-gu, Seoul, 06591, Republic of Korea. Tel: + 82-2-2258-7480 Fax: + 82-2-535-7481 E-mail: \\ sukklee@catholic.ac.kr \\ (C) The author(s). This is an open access article distributed under the terms of the Creative Commons Attribution License (https://creativecommons.org/licenses/by/4.0/). \\ See http://ivyspring.com/terms for full terms and conditions.
}

Received: 2019.05.12; Accepted: 2019.12.18; Published: 2020.01.14

\begin{abstract}
Although Epstein-Barr virus (EBV) is known to encode over 40 different miRNAs of its own, the roles of most EBV miRNAs remain unknown. Disabled homolog 2 (DAB2) is a putative tumor suppressor, but its role in gastric carcinoma (GC), especially in EBV-associated GC, needs to be clarified. Our qRT-PCR and mRNA microarray results showed that DAB2 expression was down-regulated in EBV-positive GC cells compared to EBV-negative cells. Four BART miRNAs that might target DAB2 were predicted, and we found, using a luciferase reporter assay, that miR-BART1-3p directly targeted the 3'-UTR of DAB2. The miR-BART1-3p transfection decreased DAB2 expression at both mRNA and protein levels, while transfection of an inhibitor of miR-BART1-3p, miR-BART1-3p(i), increased DAB2 expression. In addition, miR-BART1-3p as well as siDAB2 increased migration and decreased apoptosis. Meanwhile, miR-BART1-3p(i) or pcDNA3.1-DAB2 transfection decreased migration and increased apoptosis in EBV-infected GC cells. Furthermore, decreased migration by miR-BART1-3p(i) was abrogated by co-transfected siDAB2, while decreased migration by miR-BART1-3p(i) was further suppressed by a co-transfected DAB2 over-expression vector. Our data suggest that miR-BART1-3p plays an important role in the tumorigenesis of EBV-associated GC by directly targeting DAB2.
\end{abstract}

Key words: Epstein-Barr virus, BART miRNA, DAB2, cell proliferation, cell migration

\section{Introduction}

Gastric carcinoma (GC) is the third most common cause of death ( $8.2 \%$ of all cancer deaths), and the fifth most frequently diagnosed cancer [1]. To improve diagnosis or therapeutic intervention, GC has been classified in several different ways $[2,3]$. With the development of genomic technology and high-throughput sequencing, a new classification system for GC was proposed recently [4]. GC cases are divided into four subgroups based on their genomic and molecular characteristics: Epstein-Barr virus (EBV)-infected, microsatellite instable (MSI), genomically stable (GS), and chromosomal instable (CIN) [4]. EBV-associated GC (EBVaGC) comprises about 9 to $10 \%$ of all GC cases, and this proportion is not significantly different worldwide [5].

More than $90 \%$ of human populations around the world are known to be infected with EBV. EBV establishes lifelong infection in the host, indicating that EBV may counteract the effects of host immune surveillance [6, 7]. Multiple studies have demonstrated that EBV is closely associated with several tumors, including GC, Burkitt's lymphoma, nasopharyngeal carcinoma (NPC), and Hodgkin's disease. Following infection, the EBV genome is maintained extrachromosomally as an episome, and replicates using cellular replication machinery [8-10]. EBVaGC expresses a restricted number of EBV latent genes, including EBV-encoded small RNA (EBER)-1 
and -2, EBV nuclear antigen (EBNA)-1, latent membrane protein-2A (LMP2A), and BART miRNAs [11]. The expression of EBNA-1 decreased p53 activation by the loss of promyelocytic leukemia nuclear bodies, resulting in an impaired response to DNA damage and consequently promoting cell survival in EBVaGC cells [12]. LMP2A increased survivin by activating the nuclear factor- $\mathrm{kB}$ (NF- $\mathrm{kB}$ ) pathway for resistance from serum deprivation-induced apoptosis in GC cells [13]. At least 44 mature BART miRNAs have been identified [14]. BART miRNAs are expressed at high levels in all EBV-associated carcinomas such as NPC and GC. Accumulating evidence has revealed that several BART miRNAs play important roles in carcinoma development [14, 15]. However, the roles of most BART miRNAs in tumorigenesis still require further investigation.

Disabled homolog 2 (DAB2) encodes a mitogen-responsive phosphoprotein that contains a phosphotyrosine-binding domain and a proline-rich domain involved in the regulation of clathrinmediated endocytosis as well as receptor-mediated signaling pathways [16-20]. DAB2 has been considered as a putative tumor suppressor in NPC [21, 22] as well as ovarian [23, 24], breast [25-27], prostate [28], and lung carcinoma [29, 30]. Several human miRNAs have been reported to target DAB2, causing tumor promotion. Up-regulated miR-187 suppressed $\mathrm{DAB} 2$ and promoted cell proliferation [24]. The miR-93-directed down-regulation of DAB2 was shown to be the most important element that contributed to lung cancer tumorigenesis [30]. In addition, miR-93 promoted cell growth and invasion in NPC [22]. High expression of miR-106b was found in cervical cancer, and miR-106b promoted TGF- $\beta 1$-induced cervical cancer cell migration by targeting DAB2 [31]. Further, miR-191 promoted the cellular viability of estrogen receptor-positive breast cancer cells by directly suppressing the expression of DAB2 [25]. Additionally, the induction of DAB2 expression reduced cancer growth, migration, and invasion [21-25, 27-31].

However, there are few published studies showing DAB2 expression and function in EBVaGC. In this study, we observed reduced DAB2 expression in EBV-positive GC cells and investigated the EBV miRNAs involved in DAB2 down-regulation to clarify the roles of EBV BART miRNAs in EBVaGC.

\section{Materials and Methods}

\section{Cell cultures and reagents}

MKN-1, MKN-28, NCI-N87, SNU-216, and AGS are EBV-negative GC cell lines, while SNU-719,
YCCEL1, and AGS-EBV are EBV-positive GC lines. The GC cell lines were cultured in RPMI-1640 containing $10 \%$ fetal bovine serum, $100 \mathrm{U} / \mathrm{ml}$ penicillin, and $100 \mu \mathrm{g} / \mathrm{ml}$ streptomycin except for YCCEL1, which was cultured in Eagle's minimal essential medium. AGS-EBV is a GC cell line derived from AGS cells by infecting them with a recombinant Akata virus. To culture AGS-EBV cells, $400 \mu \mathrm{g} / \mathrm{ml}$ of G418 (Gibco, Carlsbad, CA, USA) was added to the medium. The human embryonic kidney cell line HEK293T was cultured in Dulbecco's modified Eagle's medium (DMEM) supplemented with 10\% FBS, $100 \mathrm{U} / \mathrm{ml}$ penicillin, and $100 \mu \mathrm{g} / \mathrm{ml}$ streptomycin. All cells were incubated at $37^{\circ} \mathrm{C}$ and supplemented with $5 \% \mathrm{CO}_{2}$.

\section{Microarray analysis}

Microarray analysis results obtained previously [32] were used for analysis. The relative expressions of tumor suppressor genes including DAB2 were assessed in AGS-EBV cells by comparing the values with those in AGS cells.

\section{Target prediction}

The DAB2 sequence used for miRNA target prediction was extracted from the National Center for Biotechnology Information (NM_001244871.1; Bethesda, MD, USA). To determine whether the 3'-UTR of DAB2 can be targeted by EBV BART miRNAs, we used a publicly available RNA hybrid program (http:// bibiserv.techfak.uni-bielefeld.de/ rnahybrid/), which is a tool for finding the minimum free energy hybridization of miRNAs to target RNAs.

\section{Transfection of miRNA mimics and miRNA inhibitors}

All of the BART miRNA mimics and the scrambled control were purchased from Genolution Pharmaceuticals (Seoul, South Korea). The locked nucleic acid (LNA) inhibitor against miR-BART1-3p and the negative-control LNA-miRNA inhibitor were purchased from Exiqon (Vedbaek, Denmark). The mirVana ${ }^{\mathrm{TM}}$ miRNA inhibitor for miR-BART1-3p and the control inhibitor were purchased from Invitrogen (Carlsbad, CA, USA). Two inhibitors showed comparable effects under the conditions used in our experiments. All transfection experiments were performed using Lipofectamine 2000 (Invitrogen) according to the manufacturer's protocol. Protein and RNA were extracted $48 \mathrm{~h}$ after transfection.

\section{Plasmid construction}

The full-length 3'-UTR of DAB2 was amplified from the cDNA of AGS-EBV cells. The amplicon was then cloned into the XhoI/NotI sites between the Renilla luciferase-coding sequence and the poly(A) 
site of the psiCHECK-2 plasmid (Promega, Madison, WI, USA) to produce psiC_DAB2. The primers used for the amplification were as follows for DAB2: 5'-TCTAGGCGATCGCTCGAGATTCTGAACTTGGT CTGCAG-3' and 5'-TTATTGCGGCCAGCGGCCG CATTCTGCCACTCCAGTTTATT-3'. Mutations were introduced into the seed sequence of psiC_DAB2 to produce psiC_DAB2m using an EZchange site-directed mutagenesis kit (Enzynomics, Daejeon, South Korea). The primers used for this purpose were as follows: 5'-CGATATTTGGGGTCATGCTAGGC CT-3' and 5'-ACGTAATGTGTTTGGCACAATCAC ATTTAGC-3'.

\section{DAB2 over-expression vector}

The DAB2 expression vector (pcDNA3.1-DAB2) constructed by $\mathrm{Du}$ et al. [30] was used to over-express DAB2 in AGS-EBV cells.

\section{Luciferase reporter assay}

To investigate the effect of miR-BART1-3p upon the expression of DAB2, HEK293T cells or AGS cells seeded in a 96-well plate $\left(5 \times 10^{3}\right.$ cells/well) were used. After $24 \mathrm{~h}$, the cells were co-transfected with $20 \mathrm{ng}$ psiC_DAB2 and $20 \mathrm{nM}$ miR-BART1-3p or with a seed sequence-mutated miR-BART1-3p (miR-BART1$3 \mathrm{pm})$. Luciferase activity was measured at $48 \mathrm{~h}$ post-transfection using a Dual-Glo luciferase reporter assay system (Promega). For each sample, Renilla luciferase activity was normalized using firefly luciferase activity.

\section{Quantitative reverse transcription PCR (qRT-PCR) for DAB2}

AGS or AGS-EBV cells were harvested, and the total RNA was extracted using the RNAiso Plus reagent (TaKaRa, Tokyo, Japan) according to the manufacturer's instructions. Next, cDNA was synthesized using $3 \mu \mathrm{g}$ total RNA, oligo(dT) primer (Macrogen, Seoul, South Korea), and Moloney murine leukemia virus (M-MLV) reverse transcriptase (Invitrogen). Real-time PCR for the indicated genes was carried out using a TOPreal ${ }^{\mathrm{TM}}$ Qpcr 2x Pre MIX SYBR-Green kit (Enzynomics, Daejeon, Korea) with the real-time PCR system (CFX96, BioRad, Hercules, CA, USA). The sequences of the primers were as follows: for DAB2, 5'-ATCCTGATCCTTTCCGT GAC-3' and 5'-TCAGCGGAGTAGACGAGCTA-3'; for GAPDH (the glyceraldehyde-3-phosphate dehydrogenase gene), 5'-ATGGGGAAGGTGAAGG TCG-3' and 5'-GGGGTCATTGATGGCAACAATA-3'. PCR conditions were $95^{\circ} \mathrm{C}$ for $10 \mathrm{~min}$, followed by 35 cycles at $95^{\circ} \mathrm{C}$ for $10 \mathrm{~s}, 60^{\circ} \mathrm{C}$ for $30 \mathrm{~s}$, and $72{ }^{\circ} \mathrm{C}$ for 30 $\mathrm{s}$. To confirm the specific amplification of the PCR product, dissociation curves were checked routinely. For this, reaction mixtures were incubated at $95^{\circ} \mathrm{C}$ for
$60 \mathrm{~s}$ and then ramped from 60 to $95^{\circ} \mathrm{C}$ at a heating rate of $0.1{ }^{\circ} \mathrm{C} / \mathrm{s}$, with fluorescence measured continuously. Relative gene expression was calculated using the quantification cycle $(\mathrm{Cq})$ values, using GAPDH as an internal standard.

\section{Quantitative reverse transcription PCR for miRNA analysis}

The miRNA cDNA was synthesized using a Mir-X miRNA First-Strand synthesis kit (Clontech, Mountain View, CA, USA) according to the manufacturer's instructions. Real-time quantitative PCR procedures were performed using a TOPreal ${ }^{\mathrm{TM}}$ Qpcr 2x Pre MIX SYBR-Green kit (Enzynomics, Daejeon, Korea). The forward primer used for miR-BART1-3p amplification was 5'-TAGCACCGC TATCCACTATGTC-3'. All amplifications were performed in triplicate, and $\mathrm{Cq}$ values were normalized to the value for an endogenous control, U6, which was supplied in the kit.

\section{Knocking down of DAB2 expression using small interfering RNA (siRNA)}

A small interfering RNA (siRNA) specific for DAB2 (siDAB2) and a control siRNA lacking any known target gene product were synthesized by Genolution Pharmaceuticals (Seoul, South Korea). The sequence of the control siRNA was 5'-CCUCGUGCCGUUCCAUCAGGUAGUU-3'. The sequence of the $\operatorname{siDAB}$ was $5^{\prime}$-GGAGUGAGGCC CUAAUGAUUU- $3^{\prime}$. AGS-EBV cells $\left(1 \times 10^{6}\right.$ cells/dish $)$ were transfected with $20 \mathrm{nM}$ siRNA using Lipofectamine 2000 (Invitrogen) in 100-mm-diameter dishes. Cells were harvested to analyze DAB2 expression $48 \mathrm{~h}$ after transfection.

\section{Western blot analysis}

Cell lysate in radioimmunoprecipitation assay (RIPA) buffer containing protease inhibitors $(1 \mathrm{mM}$ phenylmethylsulfonyl fluoride, $10 \mu \mathrm{g} / \mathrm{ml}$ leupeptin, $10 \mu \mathrm{g} / \mathrm{ml}$ pepstatin $\mathrm{A}$, and $10 \mu \mathrm{g} / \mathrm{ml}$ aprotinin) was mixed with $5 \times$ loading buffer (Fermentas, Waltham, MA, USA) and heated at $95{ }^{\circ} \mathrm{C}$ for $5 \mathrm{~min}$. Samples were separated electrophoretically on $8 \%$ sodium dodecyl sulfate (SDS)-polyacrylamide gels, and the separated proteins were transferred to a polyvinylidene fluoride (PVDF) membrane (Millipore, Billerica, MA, USA). Membranes were blocked and probed with: mouse anti-DAB2 (1:1,000; BD Biosciences, San Jose, CA, USA), or rabbit anti- $\beta$-actin (1:3,000; Cell Signaling Technology, Danvers, MA, USA) antibodies. Bound antibodies were detected with horseradish peroxidase (HRP)-conjugated anti-mouse or anti-rabbit secondary antibodies (Santa Cruz, Dallas, TX, USA) at 
a dilution of 1:5,000 for $45 \mathrm{~min}$ at room temperature. Protein bands were visualized using an enhanced chemiluminescence detection system (Amersham Biosciences), and the membrane was exposed to X-ray film (Agfa, Mortsel, Belgium). Anti- $\beta$-actin antibody was used to confirm that loading was comparable between gel lanes. The density of each protein band was read and quantified using ImageJ software.

\section{Propidium iodide (PI) staining}

Cells were harvested, washed with phosphate-buffered saline (PBS), and fixed in $70 \%$ ethanol at $-20{ }^{\circ} \mathrm{C}$ overnight. The cells were washed twice with PBS and then resuspended in PBS containing $10 \mu \mathrm{g} / \mathrm{ml}$ RNase A (Invitrogen) and 50 $\mu \mathrm{g} / \mathrm{ml}$ PI (Sigma-Aldrich, St. Louis, MO, USA). The distribution of cells in each phase of the cell cycle was analyzed using a FACSCalibur apparatus (BD Biosciences) as described previously [33].

\section{Annexin $\mathbf{V}$ staining}

Cells were washed with cold PBS and resuspended in $500 \mu \mathrm{l}$ of annexin $\mathrm{V}$ binding buffer (PE Annexin V Apoptosis Detection kit; BD Biosciences, San Diego, CA, USA), containing phycoerythrin (PE)-labeled annexin $\mathrm{V}$ and 7-amino-actinomycin $\mathrm{D}$ (7-AAD). Annexin $\mathrm{V}$ was used to label cells undergoing apoptosis by detecting phosphatidylserine (PS) on the outer plasma membrane, while 7-AAD was used to detect dead cells. After $10 \mathrm{~min}$ of incubation at room temperature in an area shielded from light, the specimens were analyzed by fluorescence-activated cell sorting (FACS) using a FACSCalibur apparatus (BD Biosciences), acquiring 10,000 events. Cells testing positive for annexin V and negative for 7-AAD were considered to be undergoing early apoptosis.

\section{Wound healing assays}

To study the effect of miR-BART1-3p on cell migration, cells $\left(5 \times 10^{5}\right)$ were seeded into 24-well plates and cultured to reach $90-95 \%$ confluence. The cell layer was scratched with a sterile $200-\mu l$ pipette tip through the confluent monolayer and washed with PBS to remove cell debris. Four hours later, the cells were transfected with appropriate combinations of siDAB2, LNA-miR-BART1-3p(i), and the DAB2 expression vector. The cells were then cultured in RPMI-1640 medium containing $3 \% \mathrm{FBS}$ at $37{ }^{\circ} \mathrm{C}$ in a humidified chamber with $5 \% \mathrm{CO}_{2}$. The scratched wounds were observed by Axiovert 200 (Carl Zeiss, Thornwood, NY, USA) microscope just after transfection (time 0 ) and at $24 \sim 48 \mathrm{~h}$ after transfection. Photographs were taken to evaluate the level of migration in each group of the transfected cells, and wound areas were assessed by ImageJ software. The experiments were repeated three times.

\section{Boyden chamber assay}

The transfected cells (AGS, AGS-EBV, MKN1, MKN28, SNU-719, or YCCEL1) were plated on $8.0 \mu \mathrm{m}$ pore size Boyden chambers (Transwell, Corning Life Sciences, Acton, MA, USA) in serum-free medium and $700 \mu \mathrm{L}$ of medium containing $10 \%$ FBS was added to the lower chambers. The chambers were then placed in an incubator at $37^{\circ} \mathrm{C}$ with $5 \% \mathrm{CO}_{2}$ for $24 \mathrm{~h}$. The cells remaining in the upper chamber were then carefully removed. The transwell membrane was fixed with $4 \%$ paraformaldehyde for $30 \mathrm{~min}$ and stained with $0.1 \%$ crystal violet for $10 \mathrm{~min}$. To count the fixed cells, images were captured randomly from 4 fields of vision using inverted microscope (IX70, Olympus, Tokyo, Japan). Three independent experiments were performed to confirm the reproducibility of the results.

\section{Statistical analyses}

The data were analyzed using one-way repeated-measures analysis of variance (ANOVA) or the Student's $t$-test. Curve fitting and analysis were performed using GraphPad Prism software (GraphPad Software, San Diego, CA, USA). P-values less than 0.05 were considered statistically significant. All results were expressed as means \pm standard deviations (SDs).

\section{Results}

\section{DAB2 is down-regulated in EBV-positive gastric carcinoma cells}

As DAB2 is considered a tumor suppressor in several cancers, we investigated the effect of EBV infection on the expression of DAB2 in gastric carcinoma cells. For this, we analyzed microarray data of AGS and AGS-EBV cells. Notably, DAB2 expression showed a greater reduction in EBV-infected cells (Figure 1A). To validate the array data, DAB2 expression was determined by real-time RT-PCR and Western blot. We found that DAB2 mRNA and protein levels were decreased in AGS-EBV cells by $43 \%$ and 58\%, respectively, compared to those in AGS cells (Figures 1B-D). These findings indicate that EBV may down-regulate the expression of DAB2 in EBV-associated gastric carcinoma.

We compared the steady-state levels of DAB2 mRNA and protein in EBV-negative (MKN45, NCI-N87, SNU216, AGS) and positive (AGS-EBV, SNU719, YCCEL1) gastric carcinoma cell lines. In general, EBV-negative gastric carcinoma cell lines showed higher DAB2 expression than did EBV-positive gastric carcinoma cell lines (Figures 
1E-F). When the films were exposed for a short period of time, a non-specific band was detected for MKN1 and MKN28 cells. These non-specific bands were visible for all of the tested cells when the films were exposed for a longer time period. Curiously, the SNU216 cell line did not express a detectable amount of DAB2, even though it is an EBV-negative GC cell line. The DAB2 gene may be disrupted in this cell line, but we did not investigate this further.

\section{Screening BART miRNAs that may target DAB2}

To test whether EBV miRNAs down-regulate DAB2 expression in EBV-positive cells, EBV miRNAs showing seed matches with the $3^{\prime}$-UTR of DAB2 were identified using an RNA hybrid program (http:// bibiserv.techfak.uni-bielefeld.de/rnahybrid/). Four BART miRNAs (miR-BART1-3p, miR-BART10-3p, miR-BART2-3p, and miR-BART12-3p) were selected

(A)

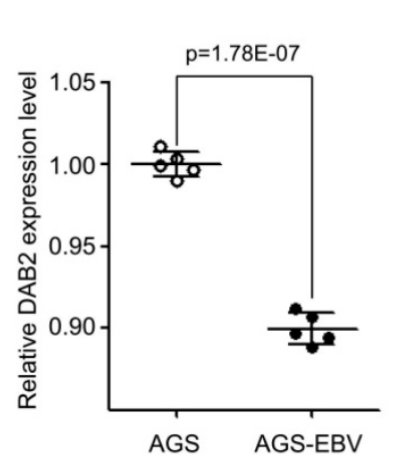

(B)

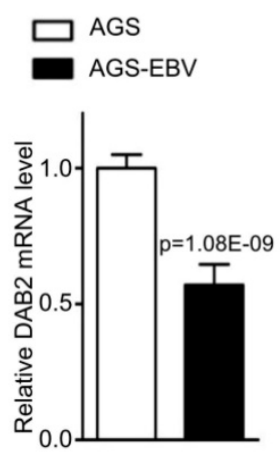

to further analyze possible interactions with the DAB2 3'-UTR (Figure 2A).

To investigate whether the BART miRNAs directly target DAB2, we constructed a luciferase reporter vector containing the entire 3'-UTR region of DAB2 (psiC_DAB2). The psiC_DAB2 reporter vector and each of the four BART miRNA mimics were co-transfected into HEK293T or AGS cells. Among the four BART miRNAs tested, only the miR-BART1-3p mimic significantly reduced luciferase activity compared to the scrambled control in both HEK293T cells (Figure 2C) and AGS cells (Figure 2D). The miR-BART2-3p and miR-BART12-3p mimics increased the luciferase activity of psiC_DAB2 (by 1.1-fold and 1.3-fold, respectively) in HEK293T cells (Figure 2C), but not in AGS cells (Figure 2D). The miR-BART10-3p did not inhibit luciferase activity in either of the cell lines. Thus, we selected miR-BART1-3p for further investigation.

(C)

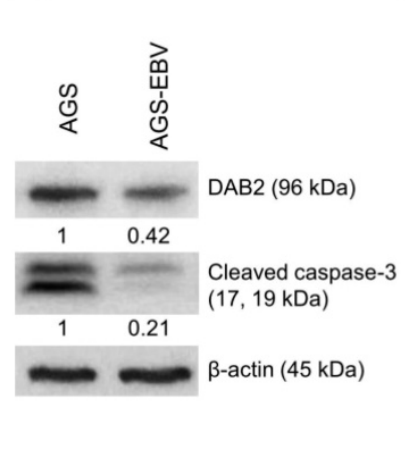

(D)

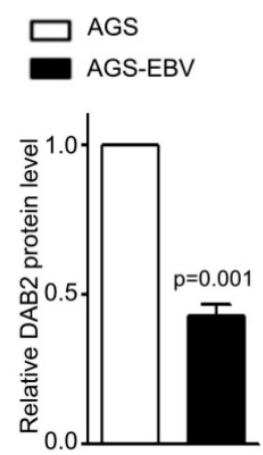

$(\mathrm{E})$

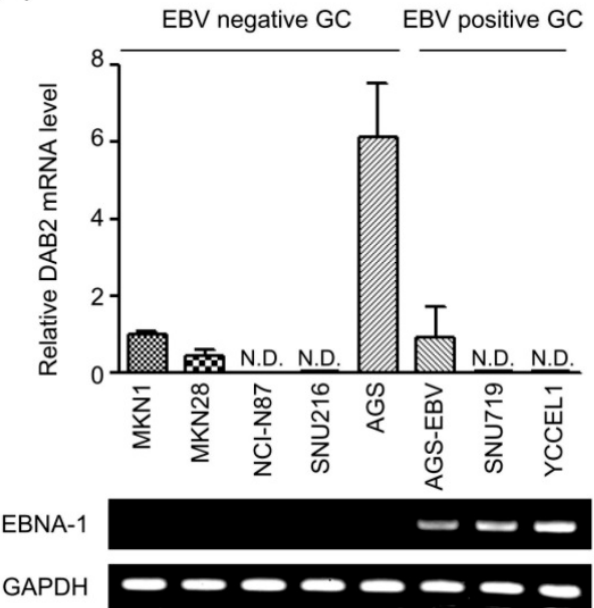

$(\mathrm{F})$

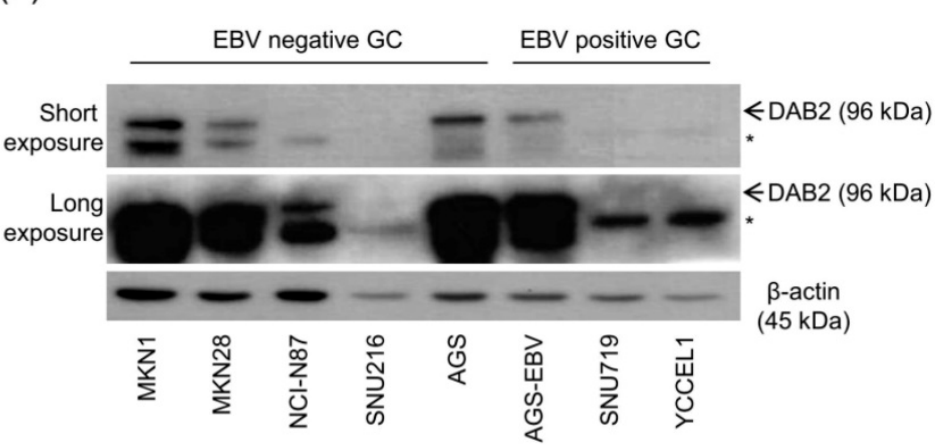

Figure 1. Reduction of DAB2 expression in EBV-infected gastric cancer cells. (A) Microarray analysis of $A G S$ and $A G S-E B V$ cells ( $n=5$ ). (B) DAB2 mRNA expression was measured by real-time RT-PCR using a SYBR Green qPCR kit. (C) DAB2 protein levels were assessed by Western blot analysis using anti-DAB2 (1:1,000) antibody. Anti- $\beta$-actin antibody was used to confirm comparable loading. (D) Western blot results similar to those shown in (C) were obtained in two more sets of independently cultured cells. The Western blot results from all three experiments have been normalized to $\beta$-actin and are expressed as ratios to the values obtained from AGS cells. Error bars indicate SD $(n=3)$. (E) Real-time RT-PCR analysis of DAB2 mRNA expression was carried out using a SYBR Green qPCR kit. EBNA-1 mRNA was used to confirm EBV infection, as it is known to be expressed in all EBV-infected cells. GAPDH was used as an internal control. (F) DAB2 protein levels were analyzed by Western blot analysis using anti-DAB2 antibody. Anti- $\beta$-actin antibody was used to confirm comparable loading. The asterisk (*) marks a non-specific band. 
(A)

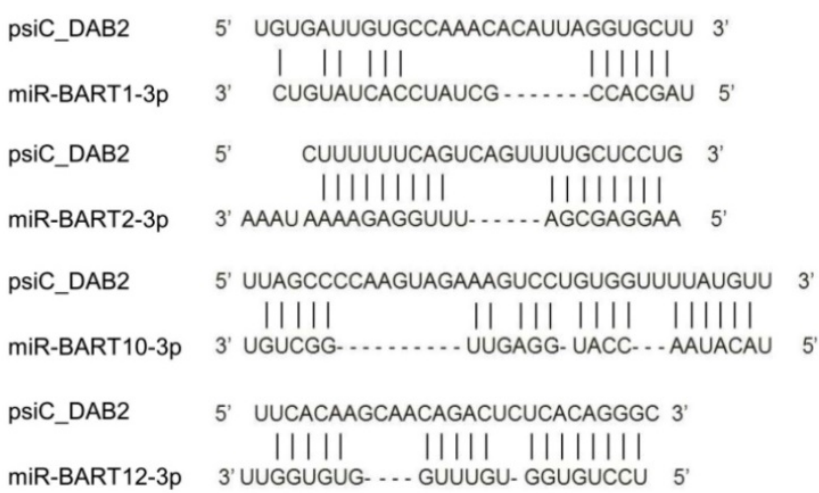

(B)

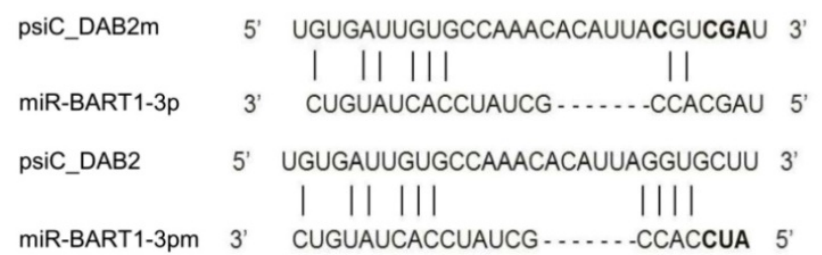

(E)

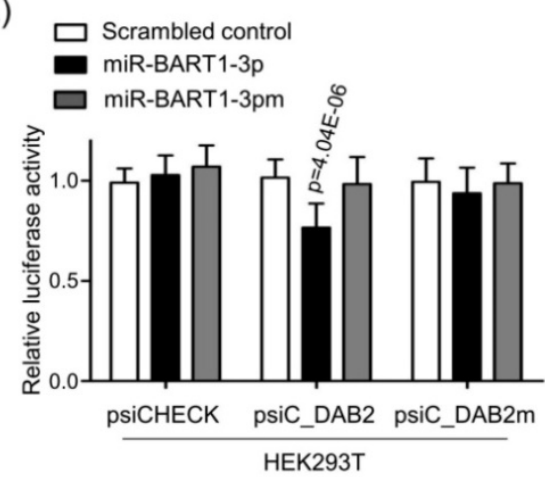

(F)
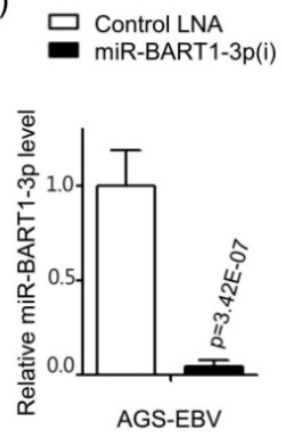

(C)
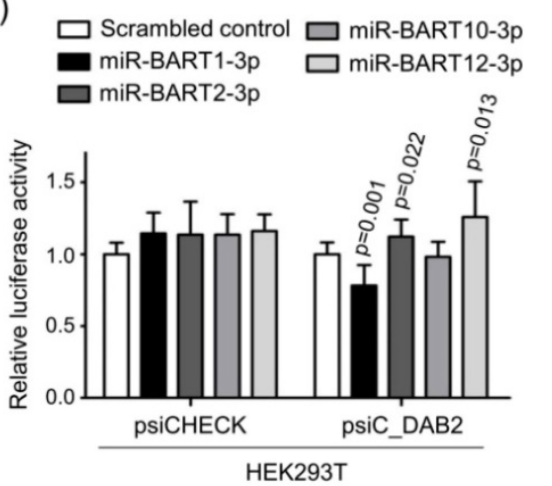

(D)
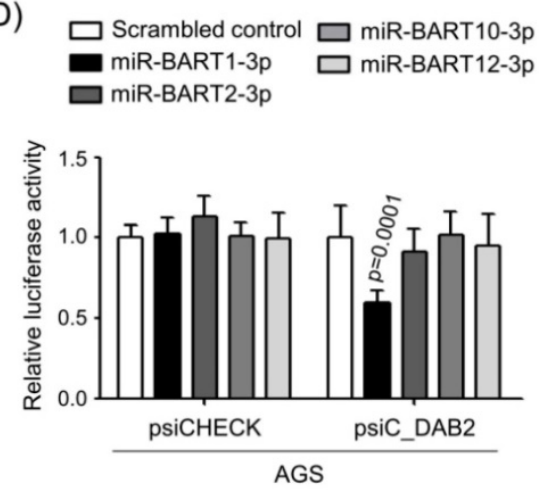

(G)
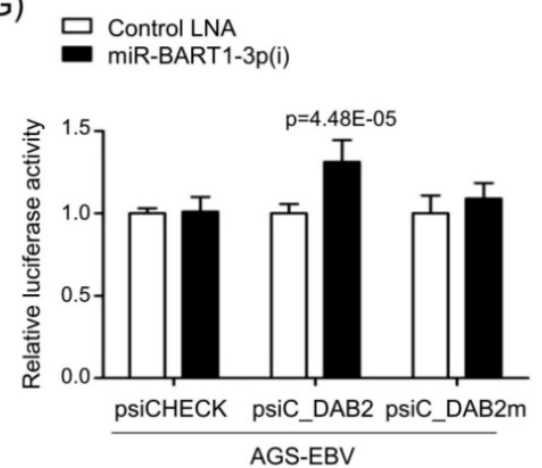

Figure 2. Screening of BART miRNAs targeting DAB2. (A) Seed match between BART miRNAs and 3'-UTR of DAB2 mRNA. (B) Seed match between miR-BART1-3p and seed match sequence-mutated (psiC_DAB2m) 3'-UTR of DAB2 mRNA (upper panel), and miR-BART1-3pm and luciferase reporter constructs containing wild type (psiC_DAB2) 3'-UTR of DAB2 mRNA (lower panel). (C-D) Luciferase activity was measured in HEK293T (C) or AGS (D) cells co-transfected with BART miRNA mimics and psiC DAB2. (E) Luciferase activity was measured in HEK293T cells co-transfected with the miR-BART1-3p mimic or seed sequence-mutated mimic (miR-BART1-3pm) and psiC_DAB2 or psiC_DAB2m. (F) Effect of miR-BART1-3p(i) on the level of miR-BART1-3p. AGS-EBV cells were transfected with 30 nM miR-BART1-3p(i). Real-time RT-PCR analysis of miR-BART1-3p expression was carried out using a SYBR Green qPCR kit. (G) Luciferase activity was measured in AGS-EBV cells co-transfected with the control inhibitor or miR-BART1-3p(i) and psiC DAB2 or psiC DAB2m. Luciferase activity was measured $48 \mathrm{~h}$ after transfection. A scrambled control and miR-BART1-3pm were used to confirm sequence-specific binding between miR-BART1-3p and the 3'-UTR of DAB2. Luciferase activity was normalized using firefly luciferase activity and expressed as a ratio to the luciferase activity obtained from the scrambled control-transfected cells. Error bars indicate SD ( $n=3)$.

\section{The miR-BART1-3p sequence specifically targets DAB2}

To test whether miR-BART1-3p targets the DAB2 sequence specifically, miR-BART1-3pm, which contains a mutation at sites $1-3$ of the seed sequence of the miR-BART1-3p (Figure 2B, bottom), was used for a luciferase reporter assay. Unlike miR-BART1-3p, the mutant mimic (miR-BART1-3pm) failed to suppress luciferase activity (Figure 2E). We also substituted sites 2, 3, 4, and 7 of the seed match sequence for miR-BART1-3p in psiC_DAB2 to prepare
psiC_DAB2m (Figure 2B, top). When psiC_DAB2m was transfected into HEK293T cells, luciferase activity was not reduced by co-transfected miR-BART1-3p (Figure 2E). These results suggest that miR-BART1-3p inhibits DAB2 expression through a sequence-specific interaction with the 3 '-UTR of DAB2 mRNA.

We then tested the effect of endogenously expressed miR-BART1-3p on DAB2 expression in EBV-infected gastric carcinoma cells using an miRNA inhibitor, miR-BART1-3p(i). The miR-BART1-3p(i) suppressed endogenously expressed miR-BART1-3p by over $90 \%$ compared to the control LNA in 
ASG-EBV cells (Figure 2F). When co-transfected into ASG-EBV cells, miR-BART1-3p(i) increased the luciferase activity of psiC_DAB2, but not that of psiC_DAB2m (Figure 2G). These results suggest that the endogenously expressed level of miR-BART1-3p in ASG-EBV was sufficient to suppress DAB2 expression by specifically targeting the 3 '-UTR of DAB2.

\section{miR-BART 1-3p regulates DAB2 expression, cell apoptosis, and migration}

To confirm the regulatory effect of miR-BART1-3p on DAB2 expression, AGS cells were transfected with miR-BART1-3p, and the mRNA level of DAB2 was analyzed by real-time RT-PCR. When transfected to AGS cells, miR-BART1-3p reduced DAB2 mRNA expression by $27 \%$ compared to the scrambled control (Figure 3A). Likewise, Western blot analysis revealed approximately $23 \%$ reduced DAB2 protein levels following miR-BART1-3p transfection (Figures 3B-C). As expected, miR-BART1-3pm failed to affect the levels of DAB2 mRNA and protein expression (Figures $3 \mathrm{~A}-\mathrm{C}$ ). These results support that the miR-BART1-3p sequence specifically down-regulates DAB2 expression.

We then investigated the biological function of miR-BART1-3p on cell death and migration ability. AGS cells were transfected with the miR-BART1-3p mimic or the scrambled control. After $48 \mathrm{~h}$, apoptotic cells were measured by FACS analysis following PI or $\mathrm{PE}$ annexin $\mathrm{V}$ staining assay (Figures 3D-F). To further investigate the effect of miR-BART1-3p on apoptosis, we analyzed apoptosis following PI staining. AGS cells transfected with miR-BART1-3p showed decreased sub-G1 cell populations (5.4 $\pm 1.8 \%)$ compared to the scrambled control-transfected AGS cells $(6.9 \pm 2.2 \%)$. miR-BART1-3p transfection increased the proportions of cells in the G0/G1 and G2/M phases, while it decreased the proportions of cells in the $S$ stage compared to scrambled control transfection (Figure 3D). Similarly, PE annexin V staining showed that apoptotic cell population (9.7 \pm $1.2 \%)$ was decreased in the miR-BART1-3p transfected AGS cells compared to the scrambled control-transfected cells $(6.2 \pm 2.0 \%)$ (Figures 3E-F). Furthermore, cleaved caspase-3, cleaved PARP, and BAX protein levels were significantly decreased in the miR-BART1-3p transfected AGS cells compared to the control cells (Figure 3G). In addition, the capability of miR-BART1-3p to reduce apoptosis induced by 5 -Fu in AGS cells was also analyzed. For this, cells were transfected with miR-BART1-3p $24 \mathrm{~h}$ prior to the 5-Fu treatment. The cells were harvested $72 \mathrm{~h}$ after transfection, and stained with PE annexin V. FACS analysis showed that miR-BART1-3p efficiently suppressed cell apoptosis induced by 5-Fu (Figure 3J-K).

We next investigated whether miR-BART1-3p could reduce DAB2 expression in some other EBV negative GC cell lines, MKN1, and MKN28 (Figure $1 F)$. In these cells, miR-BART1-3p reduced DAB2 mRNA expression compared to the scrambled control (Figure S1A). Western blot analysis revealed that DAB2 protein levels were also decreased following miR-BART1-3p transfection than following the scrambled control transfection (Figures S1B-C).

The effect of miR-BART1-3p on 5-Fu induced cell apoptosis of MKN1 and MKN28 cells was also assessed. When the cells were transfected with miR-BART1-3p, cleaved caspase-3, and cleaved PARP protein levels were significantly decreased than when the cells were transfected with the scrambled control (Figure S1D).

As several studies have reported a role of DAB2 in cell migration [34], we examined the effect of miR-BART1-3p on cell migration. Wound healing assays showed that AGS cell migration was significantly enhanced following miR-BART1-3p transfection as compared to that following the scrambled control transfection (Figures 3H-I). In addition, the Boyden chamber assay showed that miR-BART1-3p increased migration of AGS, MKN1, and MKN28 cells compared to the scrambled control (Figure S1E-F).

\section{The EBV miR-BART1-3p inhibitor blocks the effect of miR-BART 1-3p for cell apoptosis and migration in EBV-infected GC cells}

We further investigated whether an endogenously expressed level of miR-BART1-3p was sufficient to affect DAB2 expression in EBV-infected GC cells. Real-time RT-PCR and Western blot were performed to assess DAB2 levels after transfection with miR-BART1-3p(i) and the control inhibitor. In AGS-EBV cells, the DAB2 mRNA level was increased by $22 \%$ (Figure $4 \mathrm{~A}$ ), and DAB2 protein levels were enhanced by 2.2-fold following miR-BART1-3p(i) transfection compared to that following the control inhibitor transfection (Figures 4B-C). These results indicate that endogenously expressed miR-BART1-3p efficiently inhibits DAB2 expression in AGS-EBV cells.

To analyze the effect of inhibited miR-BART1-3p on cell apoptosis and migration, AGS-EBV cells were transfected with miR-BART1-3p(i) or the control inhibitor. FACS analysis following PI staining revealed that the sub-G1 population was increased $(5.4 \pm 2.0 \%)$ in miR-BART1-3p(i) transfected AGS-EBV cells compared to the control inhibitor-transfected cells $(4.6 \pm 2.0 \%)$. 
(A)

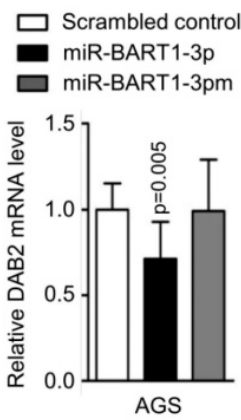

(D)

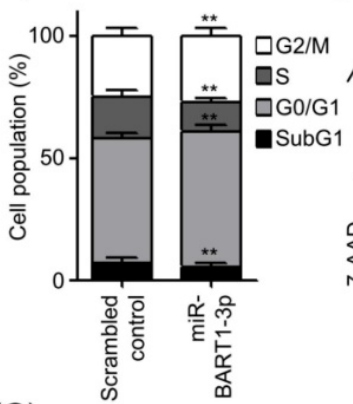

(G)

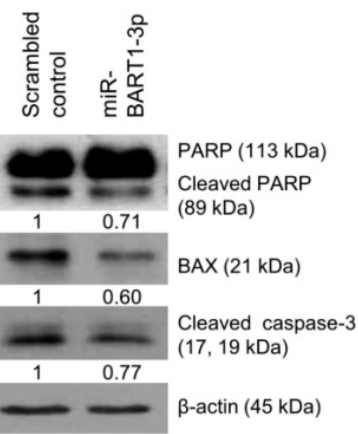

(B)

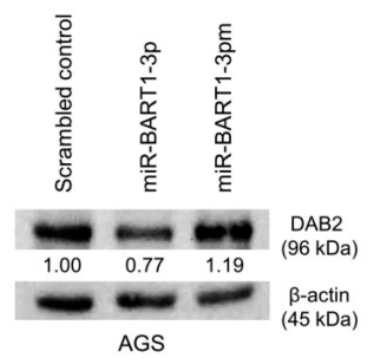

(C)
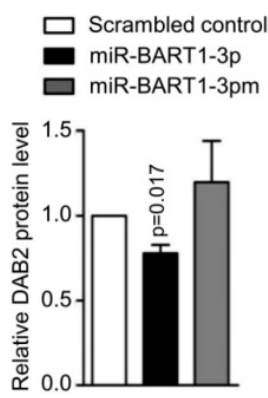

(F)
(E)

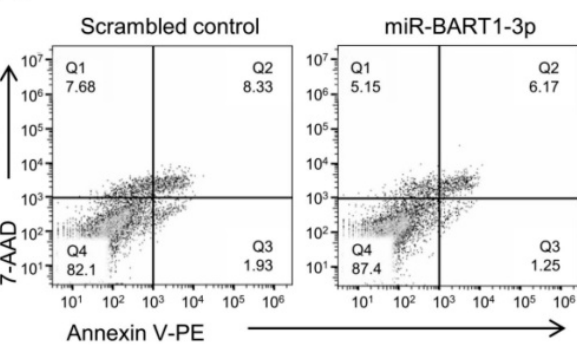

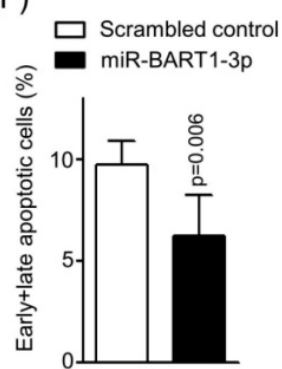

(H)

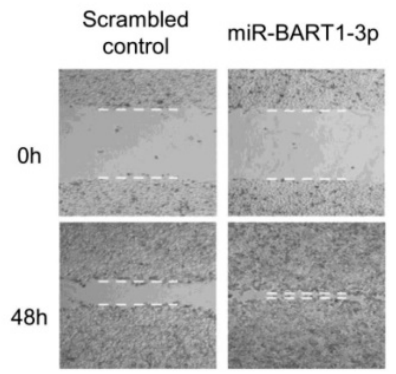

(I)

$$
\square \text { Scrambled control }
$$

miR-BART1-3p

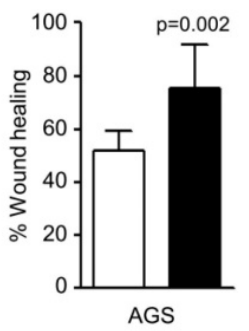

(J)

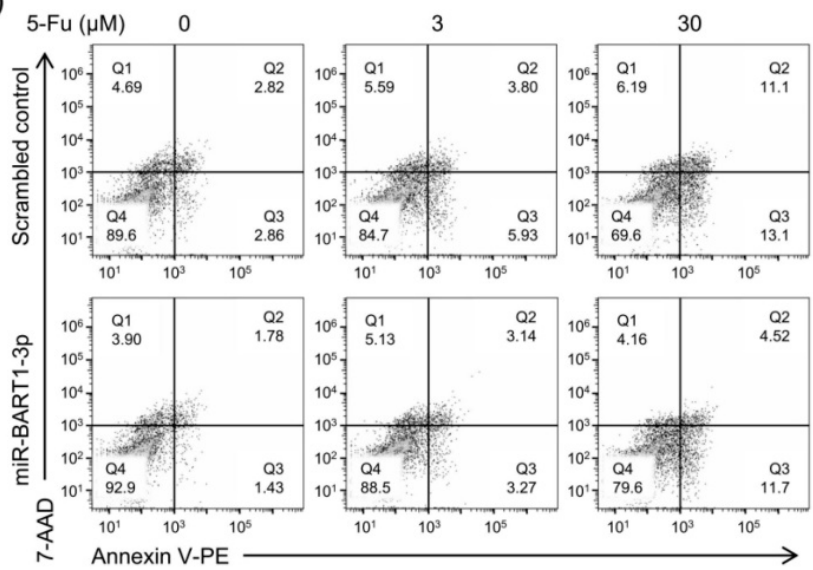

(K)

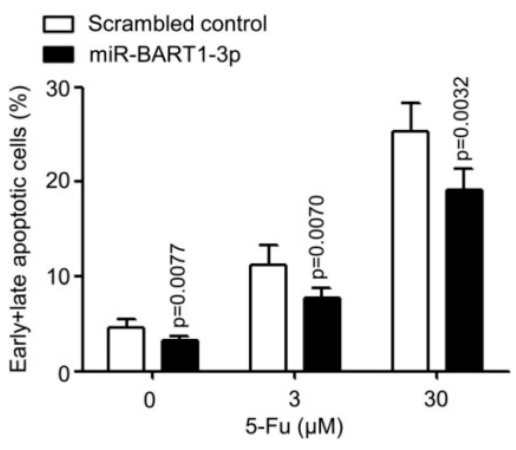

Figure 3. Effect of miR-BART1-3p on DAB2 expression in AGS cells. AGS cells were transfected with $30 \mathrm{nM}$ of miR-BART1-3p mimics, miR-BART1-3pm, or the scrambled control. (A) Real-time RT-PCR analysis of DAB2 mRNA expression was carried out using a SYBR Green qPCR kit. (B) DAB2 protein levels were analyzed by Western blot analysis using anti-DAB2 antibody. Anti- $\beta$-actin antibody was used to confirm comparable loading. (C) Western blot results similar to those shown in (B) were obtained in two more sets of independently transfected AGS cells. The Western blot results from all three experiments have been normalized to $\beta$-actin and are expressed as ratios to the values obtained from the control. (D) Cell cycle analysis was assessed by PI staining $48 \mathrm{~h}$ after the cells were transfected with miR-BART1-3p or the scrambled control. The means $\pm S D$ values from three independent experiments are plotted (**, $p<0.01)$. (E) Cells were transfected with the miR-BART1-3p mimic or the scrambled control. After $48 \mathrm{~h}$, the proportions of apoptotic cells were evaluated by FACS analysis following PE annexin $V$ staining. (F) Results similar to those in panel (E) were obtained in two more independent experiments, and the mean \pm SD values from all three independent experiments are plotted. (G) Apoptosis was assessed by cleaved PARP, BAX, and cleaved caspase- 3 protein levels by Western blot analysis. Anti- $\beta$-actin antibody was used to confirm comparable loading. $(H)$ Wound healing assays were performed to evaluate the effects of miR-BART1-3p on cell migration in AGS cells. (I) Wound width between the wound edges was evaluated using ImageJ software. The ratios of wound closure compared to the initial wound area from three independent experiments are shown as bar graphs. (J) Cells were transfected with miR-BART1-3p mimic or the scrambled control. Twenty four hours after transfection, the cells were treated with 5 -Fu $(0,3$, or $30 \mu \mathrm{M})$ for $72 \mathrm{~h}$ to induce cell apoptosis. The proportions of apoptotic cells were evaluated by FACS analysis following PE annexin $V$ staining. (K) Results similar to shown in panel $(J)$ were obtained in two more independent experiments, and the mean \pm SD values from all three experiments are plotted. Error bars indicate SD $(n=3)$. 
(A)

D. Control inhibitor

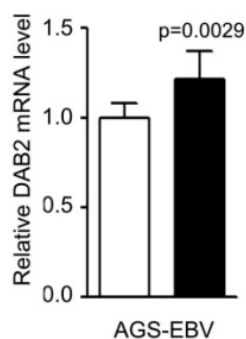

(D)

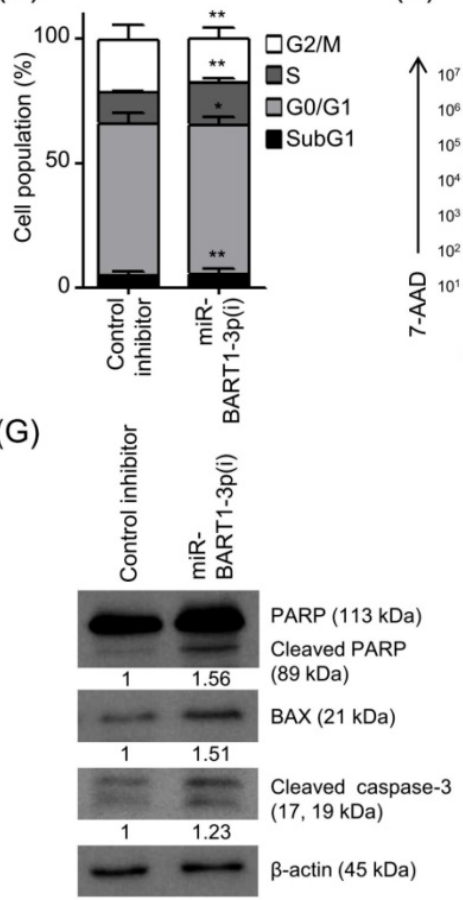

(B)

E)
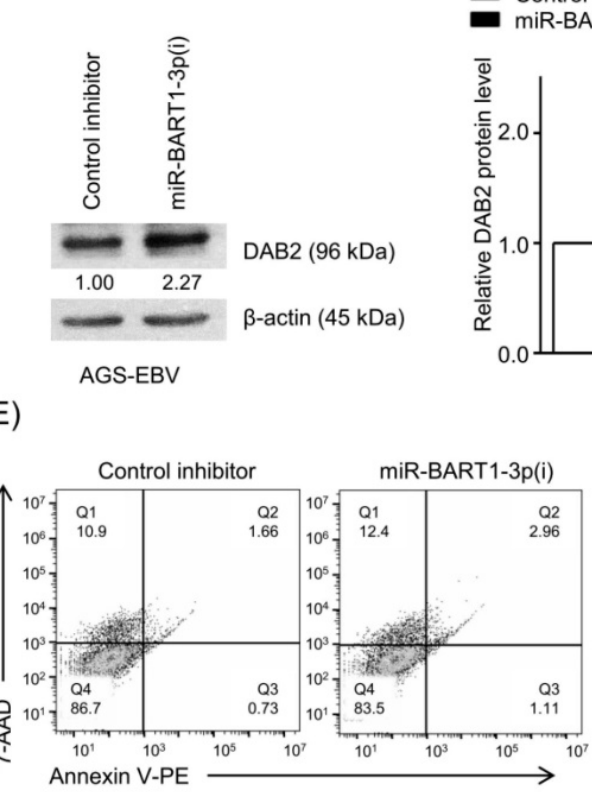

(H)

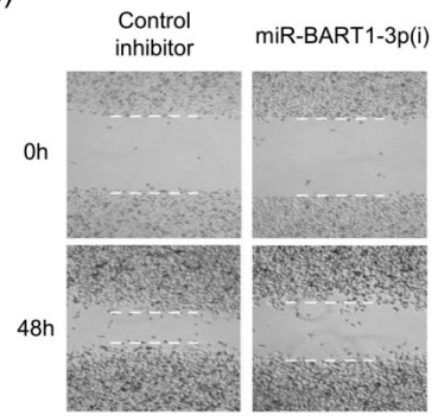

(C)

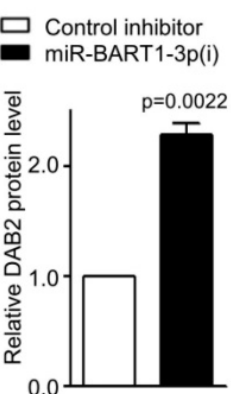

(F)

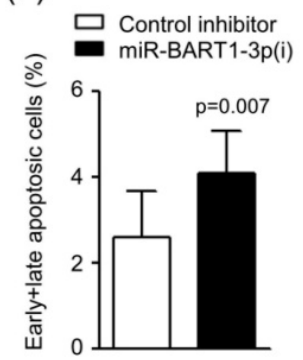

(J)

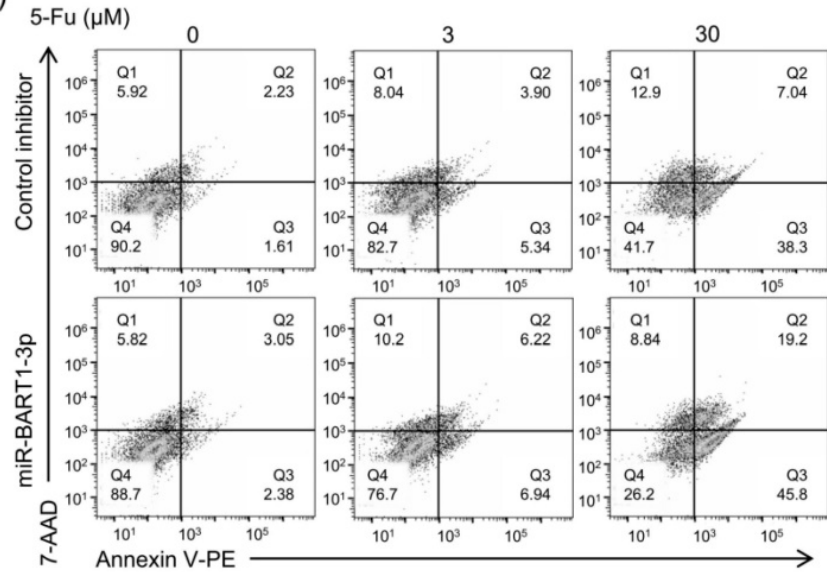

(K)

(I)

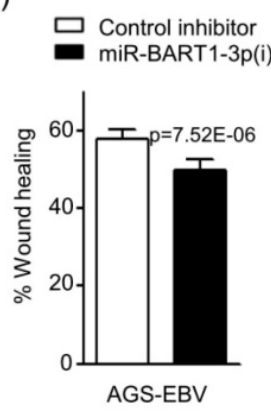

Figure 4. Effect of miR-BART1-3p(i) in AGS-EBV cells. AGS-EBV cells were transfected with $30 \mathrm{nM}$ miR-BART1-3P(i) or the control inhibitor. (A) Real-time RT-PCR analysis of DAB2 mRNA expression was carried out using a SYBR Green qPCR kit. (B) DAB2 protein levels were analyzed by Western blot analysis using anti-DAB2 antibody. Anti- $\beta$-actin antibody was used to confirm comparable loading. (C) Western blot results similar to those shown in (B) were obtained in two more sets of independently transfected AGS-EBV cells. The Western blot results have been normalized to $\beta$-actin and are expressed as ratios to the values obtained from the control. (D) Cell cycle analysis was assessed by PI staining $48 \mathrm{~h}$ after the cells were transfected with miR-BART1-3p(i) or the control inhibitor. The means \pm SD values from three independent experiments are plotted (*, $\mathrm{p}<0.05 ; * *, p<0.01$ ). (E) Cells were transfected with miR-BART1-3p(i) or the control inhibitor. After $48 \mathrm{~h}$, the proportions of apoptotic cells were assessed by PE annexin $V$ staining. (F) Results similar to those in panel (E) were obtained in two more independent experiments, and the means \pm SD from all three independent experiments are plotted. (G) Apoptosis was assessed by cleaved PARP, BAX, and cleaved caspase-3 protein levels by Western blot analysis. Anti- $\beta$-actin antibody was used to confirm comparable loading. $(\mathrm{H}) \mathrm{A}$ wound healing assay was performed to evaluate the effects of miR-BART1-3p(i) on cell migration in AGS-EBV cells. (I) Wound width between the wound edges was evaluated using Imagel software. The relative ratios of wound closure compared to the initial wound area from three independent experiments are shown as bar graphs. (J) Cells were transfected with miR-BART1-3p(i) or the control inhibitor. Twenty four hours after transfection, the cells were treated with 5 -Fu $(0,3$, or $30 \mu M)$ for $72 \mathrm{~h}$ to induce cell apoptosis. The proportions of apoptotic cells were evaluated by FACS analysis following PE annexin $V$ staining. (K) Results similar to shown in panel (J) were obtained in two more independent experiments, and the mean \pm SD values from all three experiments are plotted. Error bars indicate SD ( $n=3$ ). 
miR-BART1-3p(i) transfection decreased the proportions of cells in both the G0/G1 and G2/M phases, while it increased the proportions of cells in the $S$ phase compared to control inhibitor transfection (Figure 4D). PE annexin V staining showed that miR-BART1-3p(i) transfection increased the apoptotic cell ratio $(4.1 \pm 1.0 \%)$ more than the control inhibitor transfection did in AGS-EBV cells $(2.6 \pm 1.1 \%)$ (Figures 4E-F). Accordingly, the expression levels of cleaved PARP, BAX, and cleaved caspase- 3 proteins were significantly increased in miR-BART1-3p(i) transfected AGS-EBV cells compared to those in the control cells (Figure 4G). In addition, PE annexin V staining showed that apoptosis induced by $5-\mathrm{Fu}$ further increased following transfection with miR-BART1-3p(i) (Figure 4J-K).

As all the tested EBV positive cell lines expressed little DAB2, we investigated endogenous miR-BART1-3p level in these cells. Naturally EBV-infected GC cell lines, SNU-719, and YCCEL1, showed higher miR-BART1-3p expression level than artificially EBV-infected AGS-EBV cells (Figure S2A).

To investigate the role of miR-BART1-3p in EBV-positive GC cell lines, we transfected SNU-719 and YCCEL1 cells with miR-BART1-3p(i). Endogenously expressed miR-BART1-3p was dramatically suppressed by miR-BART1-3p(i) (Figure S2B). We then tested the effect of miR-BART1-3p(i) on 5 -Fu induced apoptosis of these cells. Western blot results showed that cleaved caspase- 3 and cleaved PARP protein levels were significantly increased in the miR-BART1-3p(i)-transfected SNU-719 and YCCEL1 cells compared to those in the control cells (Figure S2C).

Wound healing was suppressed in miR-BART1-3p(i) transfected AGS-EBV cells compared to that in the control inhibitor-transfected cells (Figures $4 \mathrm{H}-\mathrm{I}$ ). Likewise, the Boyden chamber assay also revealed that miR-BART1-3p(i) hindered migration of AGS-EBV, SNU-719, and YCCEL1 cells (Figure S2D-E).

\section{Knockdown of DAB2 using siRNA reduces cell apoptosis and induces cell migration in AGS and AGS-EBV cells}

To investigate whether siRNA against DAB2 (siDAB2) also leads to a phenotype similar to that achieved with miR-BART1-3p, siDAB2 was transfected into AGS-EBV cells. After $48 \mathrm{~h}$, real-time RT-PCR and Western blot analysis were performed to assess DAB2 expression levels. The siDAB2 transfection of AGS-EBV cells efficiently knocked down DAB2 mRNA (approximately 61\%) and protein (approximately 73\%) expression (Figures 5A-C). As reduction of DAB2 by miR-BART1-3p inhibited apoptosis, we inferred that DAB2 might regulate cell apoptosis. To test this possibility, we explored the role of siDAB2 in controlling cell apoptosis. The proportion of apoptotic cells was reduced in AGS-EBV cells following transfection with siDAB2 $(4.3 \pm 1.6 \%)$ than with the control siRNA $(7.1 \pm 1.2 \%)$. siDAB2 transfection increased the proportions of cells in both the G0/G1 and G2/M phases, while it decreased the proportions of cells in the $S$ phase compared to control siRNA transfected cells (Figure 5D). Furthermore, the levels of cleaved caspase- 3 and PARP were decreased more in siDAB2-transfected AGS-EBV cells than in the control cells (Figure 5B). These indicate that DAB2 causes apoptosis of AGS-EBV cells.

Wound healing and Boyden chamber assays showed that cell migration was decreased with up-regulation of DAB2 by miR-BART1-3p(i), while migration was increased by siDAB2 compared to the control group. The effect of miR-BART1-3p(i) on cell migration was abrogated by co-transfected siDAB2 (Figures 5E-H). These findings indicate that miR-BART1-3p inhibits apoptosis and promotes cell migration by directly inhibiting DAB2 in AGS-EBV cells.

We next inhibited DAB2 expression in AGS cells using siDAB2 to investigate whether knockdown of DAB2 shows similar effects as miR-BART1-3p. The siDAB2 efficiently knocked down DAB2 protein expression (approximately 77\%) in AGS cells (Figure $\mathrm{S} 3 \mathrm{~A})$. DAB2 knockdown using siDAB2 decreased the levels of cleaved caspase- 3 and cleaved PARP more than in the control cells (Figure S3B). The proportion of apoptotic AGS cells was reduced following transfection with siDAB2 $(2.4 \pm 0.9 \%)$ compared to the control siRNA $(3.8 \pm 0.5 \%)$. siDAB2 transfection increased the proportions of cells in both the G0/G1 and G2/M phases, while it decreased the proportion of cells in the $S$ phase compared to control siRNA transfection (Figure S3C). Furthermore, the wound healing and Boyden chamber assays showed that cell migration was increased following siDAB2 transfection (Figure S3D-G).

\section{DAB2 over-expression promotes cell apoptosis and inhibits cell migration in AGS-EBV cells}

To confirm the role of DAB2 in EBV-positive gastric carcinoma cells, we tested the effect of DAB2 over-expression using pcDNA3.1-DAB2 on apoptosis and cell migration in AGS-EBV cells. Western blotting was performed to evaluate DAB2 levels after transfection of pcDNA3.1-DAB2 or a control vector (pcDNA3.0). The DAB2 protein level was increased by 2-fold following pcDNA3.1-DAB2 transfection 
than following the control vector transfection (Figures 6A-B). The transient over-expression of DAB2 in AGS-EBV cells significantly increased apoptotic cells (Figures 6A and 6C) and decreased cell migration ability (Figures 6D-G). When AGS-EBV cells were co-transfected with the pcDNA3.1-DAB2 vector and miR-BART1-3p(i), migration was further enhanced than when cells were transfected with each of them alone (Figures 6D-E). The Boyden chamber assay also showed similar results (Figure 6F-G). These results may be attributable to the inhibitory effects of miR-BART1-3p on cell apoptosis and DAB2 expression, and demonstrate that the inhibitory function of DAB2 on cell migration is regulated by miR-BART1-3p.
(A)

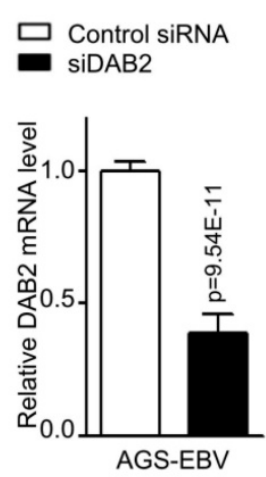

(B)

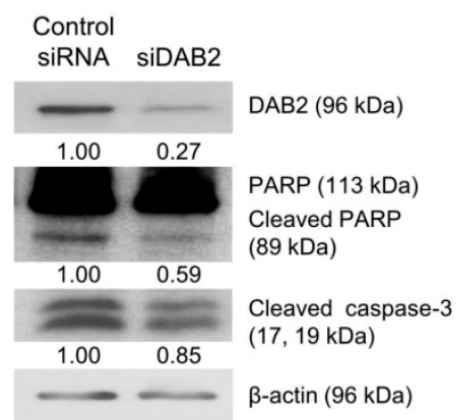

(C)

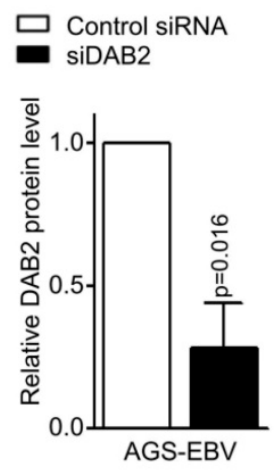

(D)

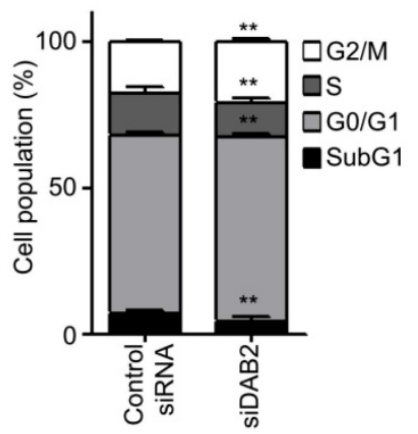

(E)

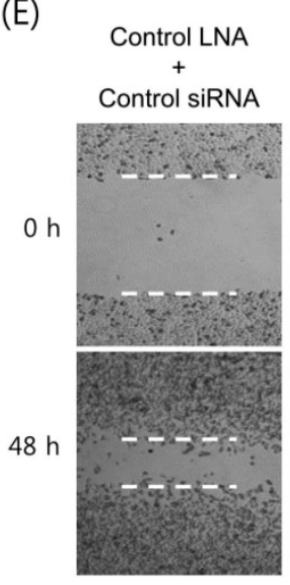

(G)

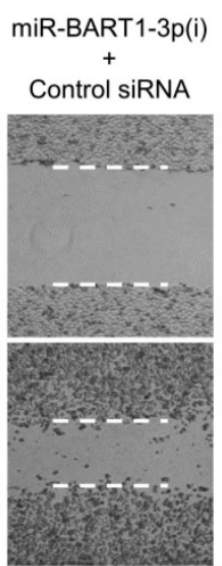

Control-LNA
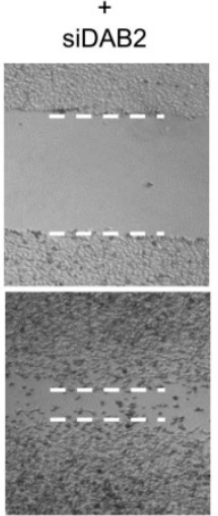

miR-BART1-3p(i) SiDAB2

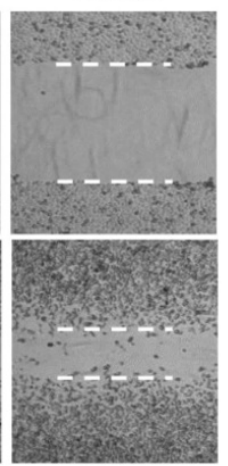

(H)

(F)
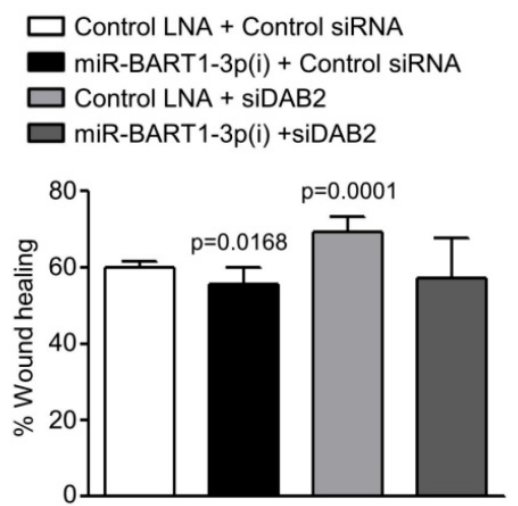
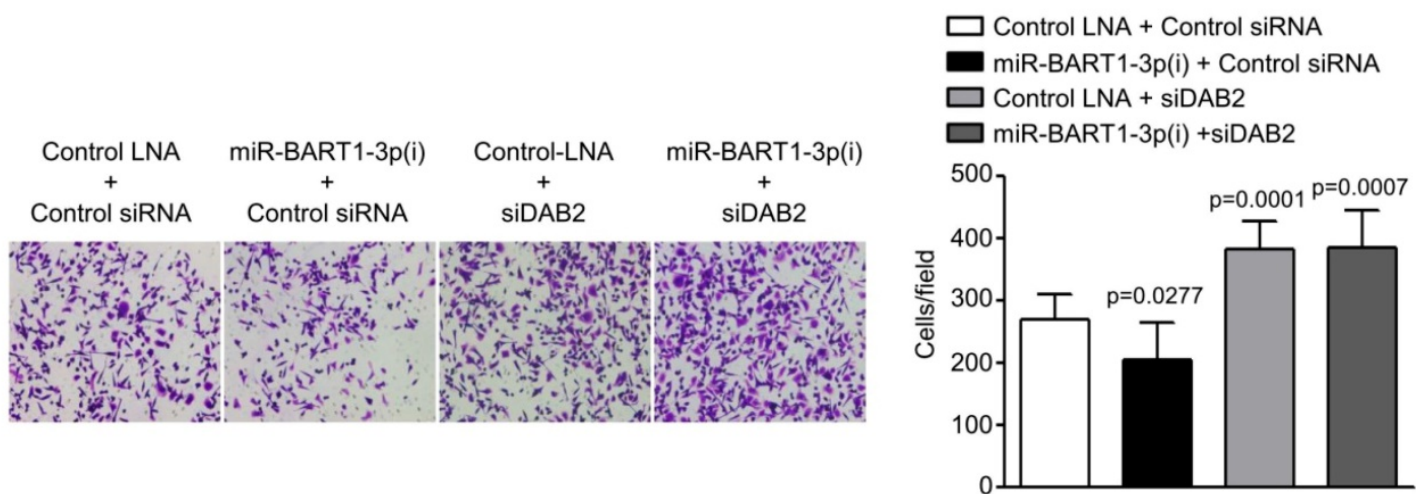

Figure 5. Effect of DAB2 knockdown using siDAB2 in AGS-EBV cells. AGS-EBV cells were transfected with $20 \mathrm{nM}$ siDAB2 or a control siRNA. (A) Real-time RT-PCR analysis of DAB2 mRNA expression was carried out using a SYBR Green qPCR kit. (B) DAB2, PARP, cleaved PARP, and cleaved caspase-3 protein levels were analyzed by Western blot analysis. Anti- $\beta$-actin antibody was used to confirm comparable loading. (C) DAB2 protein levels as shown in (B) were obtained in two more sets of independently transfected AGS-EBV cells. The Western blot results have been normalized to $\beta$-actin and are expressed as ratios to the values obtained from the control. (D) Cell cycle analysis was assessed by PI staining $48 \mathrm{~h}$ after the cells were transfected with siDAB2 or the control siRNA. The means \pm SD values from three independent experiments are plotted (**, $\mathrm{P}<0.01)$. (E) A wound healing assay was performed to evaluate the effects of miR-BART1-3p(i) and siDAB2 on cell migration in AGS-EBV cells. (F) Wound width between the wound edges was evaluated using Imagej software. The relative ratios of wound closure compared to the initial wound area from three independent experiments are shown as bar graphs. (G) Boyden chamber assays were performed to evaluate the effects of miR-BART1-3p(i) and siDAB2 on cell migration in AGS-EBV cells. (H) Results similar to shown in panel $(G)$ were obtained in two more independent experiments, and the mean \pm SD values from all three experiments are plotted. Error bars indicate $S D(n=3)$. 
(A)

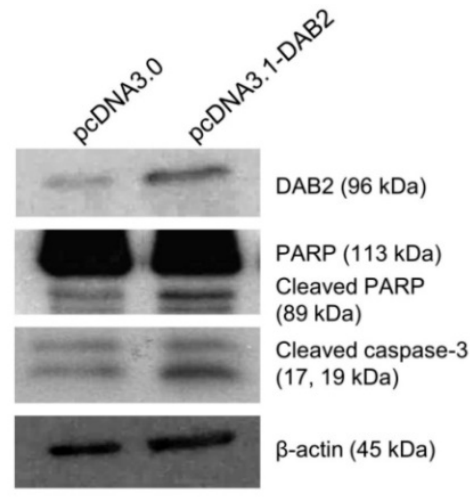

(D)

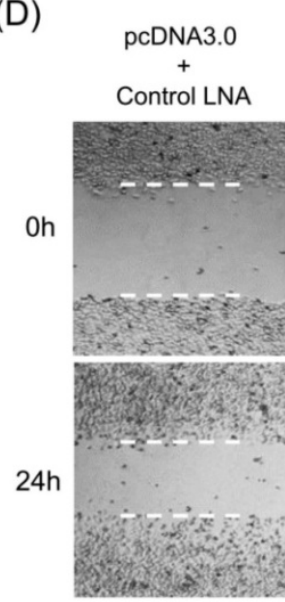

(F)

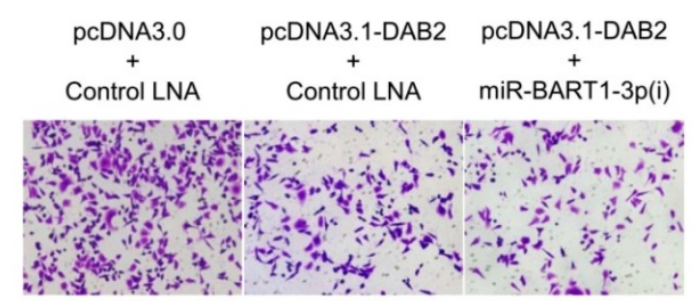

(B)
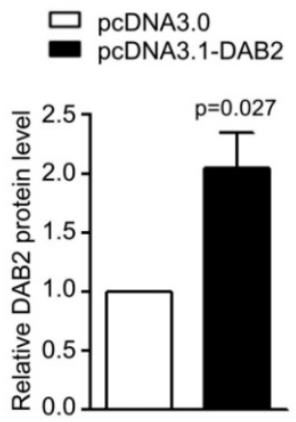

(E)
(C)

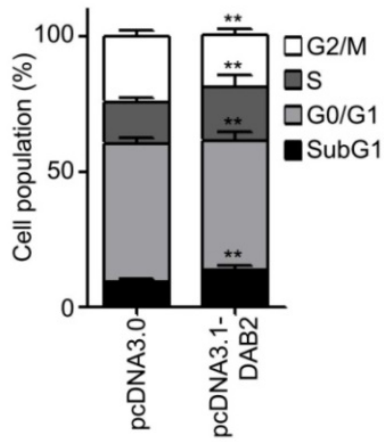

pcDNA3.0 +Control LNA
p pcDNA3.1-DAB2 +Control LNA
$\square$ pcDNA3.1-DAB2 + miR-BART1-3p(i)

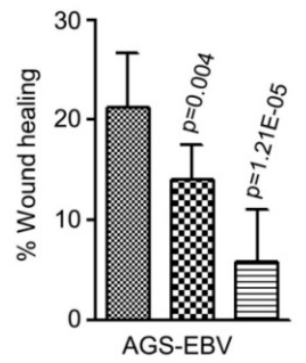

(G)

pcDNA3.0 +Control LNA

W pcDNA3.1-DAB2 +Control LNA

$\boxminus$ pcDNA3.1-DAB2 + miR-BART1-3p(i)

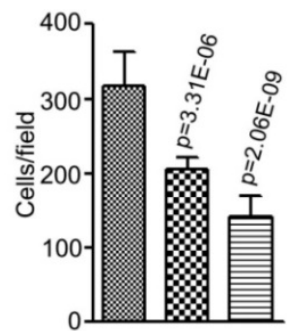

Figure 6. Effect of DAB2 over-expression in AGS-EBV cells. AGS-EBV cells were transfected with the DAB2 expression vector (pCDNA3.1-DAB2) or an empty vector (pcDNA3.0). (A) DAB2, PARP, cleaved PARP, and cleaved caspase-3 protein levels were analyzed by Western blot. Anti- $\beta$-actin antibody was used to confirm comparable loading. (B) DAB2 protein levels as shown in (A) were obtained in two more sets of independently transfected AGS-EBV cells. All three Western blot results have been normalized to the level of $\beta$-actin and are expressed as ratios to the values obtained from the control. (C) Cell cycle analysis was assessed by PI staining $48 \mathrm{~h}$ after the cells were transfected with pCDNA3.1-DAB2 or PCDNA3.0. The means + SD values from three independent experiments are plotted (**, $<<0.01$ ). (D) A wound healing assay was performed to evaluate the effects of pcDNA3.1-DAB2 and miR-BART1-3p(i) on cell migration in AGS-EBV cells. (E) Wound width between the wound edges was evaluated using ImageJ software. The relative ratios of wound closure compared to the initial wound area from three independent experiments are shown as bar graphs. (F) Boyden chamber assays were performed to evaluate the effects of DAB2 overexpression and miR-BART1-3p(i) transfection on cell migration in AGS-EBV cells. (G) Results similar to shown in panel $(F)$ were obtained in two more independent experiments, and the mean \pm SD values from all three experiments are plotted. Error bars indicate $S D(n=3)$.

\section{Discussion}

We observed low DAB2 expression in EBV-infected gastric cancer cell lines compared with EBV-negative GC cell lines. An EBV miRNA miR-BART1-3p suppressed the expression of DAB2 by specifically and directly targeting its 3'-UTR, while miR-BART1-3p(i) suppressed DAB2 expression. Transfection of miR-BART1-3p suppressed cell apoptosis and promoted cell migration. As expected, siDAB2 showed similar effects with miR-BART1-3p on apoptosis and migration. In contrast, increasing DAB2 expression by miR-BART1-3p(i) or pcDNA3.1-DAB2 transfection resulted in opposite effects on cell migration and apoptosis.

Our results showing lower DAB2 expression in EBV-positive GC cell lines than in EBV-negative cells support that DAB2 functions as a tumor suppressor in 
EBVaGC. That is consistent with previous reports showing DAB2 as a putative tumor suppressor in various cancer types [21-31]. Some EBV-negative GC cells which do not express miR-BART1-3p showed no or very little expression of DAB2. Here we did not investigate the mechanisms that down-regulated DAB2 in those cells. Perhaps other mechanisms such as promoter methylation or cellular miRNAs in addition to miR-BART1-3p, might play roles in suppressing DAB2 expression. Further studies are required to deeply understand the regulation of DAB2 in these cells.

Chen et el. [35] demonstrated that most of the highly abundant EBV miRNAs, including miR-BART1-3p, share their seed sequences with human miRNAs. Furthermore, they found that those human miRNAs were dysregulated in human malignancies, suggesting a potential linkage between highly coordinated mechanisms through which EBV miRNAs could mimic or compete with human miRNAs to affect cellular functions [35]. The miR-29 family shares a seed sequence with miR-BART1-3p. While most studies have suggested a tumor suppressor function of miR-29 with down-regulated expression in multiple human carcinoma including GC, some reports showed oncogenic functions of the miR-29 family [36-38]. Whether dysregulation of the miR-29 family sharing a seed sequence with miR-BART1-3p can be responsible for DAB2 regulation, and whether coordinated action between miR-BART1-3p and miR-29 affects viral life cycle, are questions that warrant further investigation.

We observed that miR-BART1-3p plays an important regulatory role in the apoptosis and migration of EBVaGC. Other groups have also reported oncogenic contributions of miR-BART1-3p in EBV-associated carcinomas. Cai et al. [39] demonstrated that EBV-miR-BART1 (both miR-BART1-3p and miR-BART1-5p) was highly expressed in NPC and that it increased the migration and invasion of NPC cells by directly targeting the cellular tumor suppressor PTEN. Shinozaki-Ushiku et al. [40] demonstrated that the expression level of miR-BART1-3p was highest among all of the EBV miRNAs in epithelial tumors. These findings together with our data suggest that miR-BART1-3p may play a critical role for tumorigenesis in EBVaGC.

In general, miRNA-mediated gene suppression is thought to regulate about $30 \%$ of target genes' expression [41]. However, a much larger reduction in DAB2 expression was observed in naturally EBV-infected GC cells compared to EBV-negative cells. Other mechanisms in addition to miR-BART1-3p-mediated suppression may also contribute to suppression of DAB2 expression in
EBVaGC. DAB2 promoter hypermethylation is known to be common in human malignant cancers, and there are multiple $\mathrm{CpG}$ sites in the promoter of DAB2 [21, 26, 42, 43]. EBVaGC cases show a higher prevalence of DNA hypermethylation [44]. Thus, in addition to the BART miRNA-mediated post-transcriptional regulation, DAB2 promoter hypermethylation may also contribute to low DAB2 expression in EBVaGC.

Previous observation shows that adaptor protein DAB2 is involved in multiple pathway signaling through binding motifs (c-Src [19], Wnt [20], and TGF- $\beta[45,46])$. Wong AM et al. [47] reported that significantly up-regulated EBV miRNAs may target pathway signaling such as Wnt, MAPK, and TGF- $\beta$, which are closely associated with cell cycle arrest, apoptosis, and migration. Our data suggests that the miR-BART1-3p/DAB2 axis regulates cell cycle, migration, and apoptosis presumably by modulating various signaling pathways involving DAB2 in EBV-associated GC. More delicate mechanistic studies are warranted to uncover the detailed mechanism.

In conclusion, our data suggest that EBV suppressed cell apoptosis and promoted cell migration in GC cells by miR-BART1-3p-mediated DAB2 targeting. Our findings support that DAB2 may have a tumor-suppressive function in EBVaGC.

\section{Supplementary Material}

Supplementary figures.

http://www.ijbs.com/v16p0694s1.pdf

\section{Acknowledgments}

This research was supported by Basic Science Research Program through the National Research Foundation of Korea (NRF) funded by the Ministry of Education, Science and Technology (2018R1D1A1B07041763). The DAB2 expression vector (pcDNA3.1-DAB2) was kindly provided by Dr. Alexander Pertsemlidis from the UT Health Science Center in San Antonio, Texas, USA [30].

\section{Competing Interests}

The authors have declared that no competing interest exists.

\section{References}

1. Bray F, Ferlay J, Soerjomataram I, Siegel RL, Torre LA, Jemal A. Global cancer statistics 2018: GLOBOCAN estimates of incidence and mortality worldwide for 36 cancers in 185 countries. CA: a cancer journal for clinicians. 2018; 68: 394-424

2. Waldum HL, Fossmark R. Types of Gastric Carcinomas. International journal of molecular sciences. 2018; 19.

3. Hu B, El Hajj N, Sittler S, Lammert N, Barnes R, Meloni-Ehrig A. Gastric cancer: Classification, histology and application of molecular pathology. Journal of gastrointestinal oncology. 2012; 3: 251-61.

4. Cancer Genome Atlas Research N. Comprehensive molecular characterization of gastric adenocarcinoma. Nature. 2014; 513: 202-9. 
5. Morales-Sanchez A, Fuentes-Panana EM. Epstein-Barr Virus-associated Gastric Cancer and Potential Mechanisms of Oncogenesis. Current cancer drug targets. 2017; 17: 534-54.

6. Price AM, Luftig MA. To be or not IIb: a multi-step process for Epstein-Barr virus latency establishment and consequences for B cell tumorigenesis. PLoS pathogens. 2015; 11: e1004656.

7. Wang M, Yu F, Wu W, Wang Y, Ding H, Qian L. Epstein-Barr virus-encoded microRNAs as regulators in host immune responses. International journal of biological sciences. 2018; 14: 565-76.

8. Rickinson AB. Co-infections, inflammation and oncogenesis: future directions for EBV research. Seminars in cancer biology. 2014; 26: 99-115.

9. Oh ST, Kim M, Lee SK. Maintenance of the viral episome is essential for the cell survival of an Epstein-Barr virus positive gastric carcinoma cell line. Archives of pharmacal research. 2009; 32: 729-36

10. Ghosh SK, Perrine SP, Faller DV. Advances in Virus-Directed Therapeutics against Epstein-Barr Virus-Associated Malignancies. Advances in virology. 2012; 2012: 509296.

11. Shinozaki-Ushiku A, Kunita A, Fukayama M. Update on Epstein-Barr virus and gastric cancer (review). International journal of oncology. 2015; 46: 1421-34.

12. Sivachandran N, Dawson CW, Young LS, Liu FF, Middeldorp J, Frappier L. Contributions of the Epstein-Barr virus EBNA1 protein to gastric carcinoma. Journal of virology. 2012; 86: 60-8.

13. Hino R, Uozaki $H$, Inoue $Y$, Shintani $Y$, Ushiku T, Sakatani $T$, et al. Survival advantage of EBV-associated gastric carcinoma: survivin up-regulation by viral latent membrane protein 2A. Cancer research. 2008; 68: 1427-35.

14. Bernier A, Sagan SM. The Diverse Roles of microRNAs at the Host(-)Virus Interface. Viruses. 2018; 10.

15. Catalanotto C, Cogoni C, Zardo G. MicroRNA in Control of Gene Expression: An Overview of Nuclear Functions. International journal of molecular sciences. 2016; 17: 1712 .

16. Fu L, Rab A, Tang LP, Rowe SM, Bebok Z, Collawn JF. Dab2 is a key regulator of endocytosis and post-endocytic trafficking of the cystic fibrosis transmembrane conductance regulator. The Biochemical journal. 2012; 441: 633-43.

17. Tao W, Moore R, Smith ER, Xu XX. Endocytosis and Physiology: Insights from Disabled-2 Deficient Mice. Front Cell Dev Biol. 2016; 4: 129.

18. Zhou J, Hsieh JT. The inhibitory role of DOC-2/DAB2 in growth factor receptor-mediated signal cascade. DOC-2/DAB2-mediated inhibition of ERK phosphorylation via binding to Grb2. The Journal of biological chemistry. 2001; 276: 27793-8

19. Zhou J, Scholes J, Hsieh JT. Characterization of a novel negative regulator (DOC-2/DAB2) of c-Src in normal prostatic epithelium and cancer. The Journal of biological chemistry. 2003; 278: 6936-41.

20. Hocevar BA, Mou F, Rennolds JL, Morris SM, Cooper JA, Howe PH. Regulation of the Wnt signaling pathway by disabled-2 (Dab2). The EMBO journal. 2003; 22: 3084-94.

21. Tong $\mathrm{JH}, \mathrm{Ng} \mathrm{DC}$, Chau $\mathrm{SL}$, So KK, Leung PP, Lee TL, et al, Putative tumour-suppressor gene DAB2 is frequently down regulated by promoter hypermethylation in nasopharyngeal carcinoma. BMC Cancer. 2010; 10: 253.

22. Xu YF, Mao YP, Li YQ, Ren XY, He QM, Tang XR, et al. MicroRNA-93 promotes cell growth and invasion in nasopharyngeal carcinoma by targeting disabled homolog-2. Cancer letters. 2015; 363: 146-55.

23. Mok SC, Chan WY, Wong KK, Cheung KK, Lau CC, Ng SW, et al. DOC-2, a candidate tumor suppressor gene in human epithelial ovarian cancer. Oncogene. 1998; 16: 2381-7.

24. Chao A, Lin CY, Lee YS, Tsai CL, Wei PC, Hsueh S, et al. Regulation of ovarian cancer progression by microRNA-187 through targeting Disabled homolog-2. Oncogene. 2012; 31: 764-75.

25. Tian X, Zhang Z. miR-191/DAB2 axis regulates the tumorigenicity of estrogen receptor-positive breast cancer. IUBMB life. 2018; 70: 71-80.

26. Bagadi SA, Prasad CP, Srivastava A, Prashad R, Gupta SD, Ralhan R. Frequent loss of Dab2 protein and infrequent promoter hypermethylation in breast cancer. Breast cancer research and treatment. 2007; 104: 277-86.

27. Martin JC, Herbert BS, Hocevar BA. Disabled-2 downregulation promotes epithelial-to-mesenchymal transition. Br J Cancer. 2010; 103: 1716-23.

28. Xie Y, Zhang Y, Jiang L, Zhang M, Chen Z, Liu D, et al. Disabled homolog 2 is required for migration and invasion of prostate cancer cells. Frontiers of medicine. 2015: 9: 312-21.

29. Xie XM, Zhang ZY, Yang LH, Yang DL, Tang N, Zhao HY, et al. Aberrant hypermethylation and reduced expression of disabled-2 promote the development of lung cancers. International journal of oncology. 2013; 43: 1636-42.

30. Du L, Zhao Z, Ma X, Hsiao TH, Chen Y, Young E, et al. miR-93-directed downregulation of DAB2 defines a novel oncogenic pathway in lung cancer. Oncogene. 2014; 33: 4307-15.

31. Cheng Y, Guo Y, Zhang Y, You K, Li Z, Geng L. MicroRNA-106b is involved in transforming growth factor beta1-induced cell migration by targeting disabled homolog 2 in cervical carcinoma. Journal of experimental \& clinical cancer research : CR. 2016; 35: 11.

32. Kim H, Choi H, Lee SK. Epstein-Barr virus miR-BART20-5p regulates cell proliferation and apoptosis by targeting BAD. Cancer letters. 2015; 356: 733-42.

33. Shin HJ, Kim DN, Lee SK. Association between Epstein-Barr virus infection and chemoresistance to docetaxel in gastric carcinoma. Molecules and cells. 2011; 32: 173-9.
34. Teckchandani A, Toida N, Goodchild J, Henderson C, Watts J, Wollscheid B, et al. Quantitative proteomics identifies a Dab2/integrin module regulating cell migration. The Journal of cell biology. 2009; 186: 99-111.

35. Chen SJ, Chen GH, Chen YH, Liu CY, Chang KP, Chang YS, et al. Characterization of Epstein-Barr virus miRNAome in nasopharyngeal carcinoma by deep sequencing. PloS one. 2010; 5 .

36. Kwon JJ, Factora TD, Dey S, Kota J. A Systematic Review of miR-29 in Cancer. Molecular therapy oncolytics. 2018; 12: 173-94.

37. Hwang J, Min BH, Jang J, Kang SY, Bae H, Jang SS, et al. MicroRNA Expression Profiles in Gastric Carcinogenesis. Scientific reports. 2018; 8: 14393.

38. Zhao X, Hou Y, Tuo Z, Wei F. Application values of miR-194 and miR-29 in the diagnosis and prognosis of gastric cancer. Experimental and therapeutic medicine. 2018; 15: 4179-84.

39. Cai L, Ye Y, Jiang O, Chen Y, Lyu X, Li J, et al. Epstein-Barr virus-encoded microRNA BART1 induces tumour metastasis by regulating PTEN-dependent pathways in nasopharyngeal carcinoma. Nature Communications. 2015; 6: 7353.

40. Shinozaki-Ushiku A, Kunita A, Isogai M, Hibiya T, Ushiku T, Takada K, et al. Profiling of Virus-Encoded MicroRNAs in Epstein-Barr Virus-Associated Gastric Carcinoma and Their Roles in Gastric Carcinogenesis. Journal of virology. 2015; 89: 5581-91.

41. Osada H, Takahashi T. MicroRNAs in biological processes and carcinogenesis. Carcinogenesis. 2007; 28: 2-12.

42. Sheng Z, He J, Tuppen JA, Sun W, Fazili Z, Smith ER, et al. Structure, sequence, and promoter analysis of human disabled-2 gene (DAB2). Genomics. 2000; 70: 381-6.

43. Zhang Z, Chen Y, Tang J, Xie X. Frequent loss expression of dab2 and promotor hypermethylation in human cancers: a meta-analysis and systematic review. Pak J Med Sci. 2014; 30: 432-7.

44. The Cancer Genome Atlas Research N, Bass AJ, Thorsson V, Shmulevich I, Reynolds SM, Miller M, et al. Comprehensive molecular characterization of gastric adenocarcinoma. Nature. 2014; 513: 202.

45. Prunier C, Howe PH. Disabled-2 (Dab2) is required for transforming growth factor beta-induced epithelial to mesenchymal transition (EMT). The Journal of biological chemistry. 2005; 280: 17540-8.

46. Hocevar BA, Smine A, Xu XX, Howe PH. The adaptor molecule Disabled-2 links the transforming growth factor beta receptors to the Smad pathway. The EMBO journal. 2001; 20: 2789-801.

47. Wong AM, Kong KL, Tsang JW, Kwong DL, Guan XY. Profiling of Epstein-Barr virus-encoded microRNAs in nasopharyngeal carcinoma reveals potential biomarkers and oncomirs. Cancer. 2012; 118: 698-710. 